Check for updates

New Taipei City

aesilver360@gmail.com

Cite this as: $B M J 2021 ; 374: n 2104$ http://dx.doi.org/10.1136/bmj.n2104 Published: 03 September 2021
COVID-19

\section{Why Taiwan approved its own vaccine before phase III trials}

Taiwan garnered international acclaim for its non-pharmaceutical measures to combat community transmission of covid-19. The island has now controversially fast tracked a local vaccine candidate. Andrew Silver asks why

\section{Andrew Silver freelance science writer}

On 19 July 2021, Medigen Vaccine Biologics became the first local company to win emergency approval from Taiwan's regulator for a covid-19 vaccine candidate.

But the drug's approval has sparked controversy. The emergency use authorisation was granted under an accelerated evaluation system for covid vaccine candidates, which does not require the traditional results from a phase III clinical trial on safety and efficacy that will be required for full approval. The system instead relied on data from an expanded phase II clinical trial and an immune response comparison with the AstraZeneca adenovirus vaccine. Under the approval terms Medigen will be required to monitor safety and submit a report on effectiveness within one year.

In response, a former mayor of Taipei City and a former health minister have demanded proof of clinical efficacy, and on 4 August they filed a formal request with the Taipei High Administrative Court to overturn the approval decision. The two said that the comparison made by Taiwan's Food and Drug Administration (FDA) was not sufficient to justify approval and that the island should wait for a clinical trial verifying efficacy. The court dismissed the request on 20 August, with the plaintiffs considering an appeal.

The FDA said in October 2020 that to speed up the island's vaccine access it would allow developers to apply for temporary approval if they completed a stage II clinical trial expanding the number of participants from 1300 to at least 3000. A spokesperson told The BMJ that the comparison the agency made was the best it could do to judge protection and that 18 members of its covid-19 vaccine advisory committee supported Medigen's approval (three supported, 15 supported conditionally, one was against, one wanted additional data, and the chair did not voice a vote).

The controversy reflects a dilemma facing parts of the Asia Pacific region which, before vaccines were available, had been praised for their handling of the pandemic, keeping community transmission and deaths down through border controls and mobility restrictions. Taiwan, with about 24 million people, has one of the lowest per capita rates of covid-19 worldwide. As of 1 September its total confirmed case count in the pandemic was 16 oo1, with 836 deaths. ${ }^{1}$

May 2021 saw Taiwan's first major rise in cases since the start of the pandemic, data from Taiwan's Centers for Disease Control (CDC) have shown. It recorded a total of just 1163 cases from January 2020 to April 2021, but 8917 in May 2021, followed by 4873 in June, 772 in July and 276 in August. ${ }^{23}$ To combat local transmission in case of a future outbreak that can't be controlled quickly, the island wants to boost vaccination rates quickly, but rates remain low because many people can't access vaccines.

It's the problem of global supply. Chuang Jen-Hsiang, deputy director general at Taiwan's CDC, says that production delays are restricting the supply of covid vaccines globally and delaying shipments. He told The BMJ he thought that the island would probably have administered 80 doses in every 100 people by the end of 2021. The rate as of 31 August is 46.69 doses in 100 people, although fewer than a million people are fully vaccinated with two doses.

To help give people access to safe and effective vaccines as soon as possible, authorities are procuring doses from international channels such as Covax or from vaccine manufacturers directly. Taiwan bans vaccines from the Chinese mainland, so they are not an option. And it is simultaneously looking to locally developed vaccines that could offer a source of protection for its people and an economic opportunity from overseas sales.

\section{Medigen} Taiwan's FDA approved for people aged 20 and older. The government has ordered five million doses, which the company aims to deliver by the end of 2021 but the CDC says it will not force anyone to take. Some 650830 doses have been administered since it became publicly available on 23 August, including to over 2000 people working in medical settings. The island's president, Tsai Ing-wen, was among the first to receive a dose, as shown on live TV. 45

The vaccine uses a traditional protein delivery system (as opposed to the newer mRNA or adenovirus methods of Pfizer-BioNTech, Moderna, or AstraZeneca), which the company already uses in developing other vaccines for diseases such as influenza.

Medigen conducted an expanded phase II clinical trial in Taiwan, with data from 3815 participants aged 20-89 presented to the FDA. Taiwan's FDA says that it has no major safety concerns, and the CDC states that the most common side effects are pain, general weakness, headaches, and diarrhoea. Only $0.7 \%$ of
Medigen's candidate is a two dose vaccine that 
people who receive the vaccine develop fevers (compared with 7.9\% with AstraZeneca's vaccine and 15.5\% with Moderna's).

But the question is, does it work? The FDA says that in select groups of people the levels of antibodies against the original wild-type SARS-CoV-2 strain 28 days after a second dose of Medigen were no worse than those elicited by the AstraZeneca vaccine. Taiwan's FDA told The BMJ that the two populations analysed were not identical, but it was confident that the comparison it made was valid and that Medigen should offer some protection against covid-19-a sentiment echoed by the CDC.

Medigen Vaccine Biologics' chief medical officer, Allen Lien, told The BMJ that the company could "comfortably predict that if we go to a phase III trial, our efficacy will be at least not inferior to AstraZeneca.” It's preparing to conduct a formal phase III study and a phase IV study on real world effectiveness. Lien declined to provide a timeline for the phase III study but said that it would report effectiveness data from a phase IV study within 12 months.

\section{Bridging}

Without phase III clinical trial data on efficacy the FDA has instead employed a process called "bridging," which speeds up approval for new vaccine candidates or the use of approved vaccines among different populations by comparing protective immune responses, but not everyone supports the practice for new covid vaccine candidates. "It's a controversial issue," said Nathan Chen, chief medical officer of the local vaccine developer Adimmune, which is developing its own protein covid-19 vaccine candidate and is in collaboration talks with Novavax and Sanofi.

Evidence suggests that the levels of SARS-CoV-2 blocking antibodies (which can prevent infection) that are present in early stage human clinical trials of covid-19 vaccines are good predictors of efficacy in late stage clinical trials, and they are believed to be the top candidate for bridging. But scientists don't yet fully understand the body's immune response to covid-19.

Some people worry that differences between covid vaccines could make an antibody comparison unreliable for inferring how much protection a vaccine candidate provides. "You can't compare apples and oranges," said Wu Suh-Chin, a vaccine researcher at National Tsing Hua University in Hsinchu who doesn't support the vaccine's emergency approval. "I am more traditionally minded for the vaccine efficacy determined by phase III clinical endpoints.”

Still, many in Taiwan do support the approval. Ho Mei-Shang, an epidemiologist at Academia Sinica's Institute of Biomedical Sciences in Taipei, said that for highly active younger groups who couldn't access other vaccines, having antibodies was better than having no protection at all.

Lee Ping-Ing, a paediatrician at National Taiwan University Hospital in Taipei and honorary chair of the Taiwan Immunization Vision and Strategy organisation, who convenes a CDC advisory committee on immunisation practices, said that the vaccine's approval had a strong scientific footing and that protein vaccines may be a good choice for people who were concerned about potential allergic reactions or side effects from adenovirus or mRNA vaccines.

\section{Going global}

The World Health Organization said in a statement to The BMJ, "There is an ongoing debate among regulators looking at a situation that is evolving, as placebo controlled trials will become even more difficult to perform. Being domestically developed or not is not the question. In a pandemic situation, all vaccines need to be prepared to meet internationally accepted standards in order to be used anywhere.

"Currently there are debates on how to use it, but the limited availability of comparator vaccines data makes it a challenge to meet the requirements of a strong regulatory framework and appropriately designed clinical trials.”

Medigen's Lien said that the company had followed strict rules set by Taiwan's FDA for emergency approval and that there was no single standard for vaccines that would meet the requirements of all jurisdictions around the world, but it was aiming to supply globally and was in talks with other regulators. "There's no unified global governance in a lot of issues regarding health," he said. "We live in a very divided world."

Twu Shiing-jer, a former health minister who chairs the Development Center for Biotechnology in New Taipei City, said that the judgment by the FDA's advisory committee should be respected. He told The $B M J$ that he personally supported the approval decision and believed that Taiwan's need was urgent. Medigen is very much "for emergency use,” said Twu. "Our weapon is vaccines.”

Competing interests: I have read and understood BMJ policy on declaration of interests and have no relevant interests to declare.

Provenance and peer review: Commissioned, not externally peer reviewed.

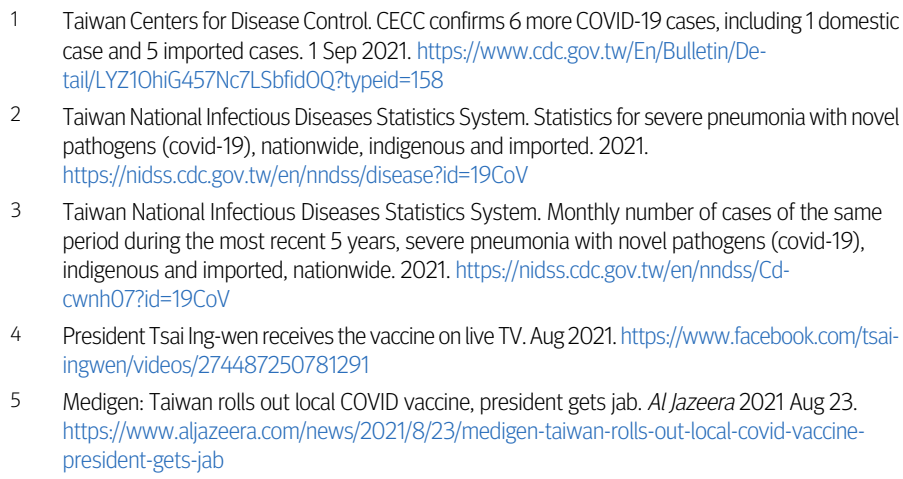

2 Taiwan National Infectious Diseases Statistics System. Statistics for severe pneumonia with novel pathogens (covid-19), nationwide, indigenous and imported. 2021.

3 Taiwan National Infectious Diseases Statistics System. Monthly number of cases of the same period during the most recent 5 years, severe pneumonia with novel pathogens (covid-19), indigenous and imported, nationwide. 2021. https://nidss.cdc.gov.tw/en/nndss/Cdcwnh07? id=19 CoV

4 President Tsai Ing-wen receives the vaccine on live TV. Aug 2021. https://www.facebook.com/tsaiingwen/videos/274487250781291

5 Medigen: Taiwan rolls out local COVID vaccine, president gets jab. Al Jazeera 2021 Aug 23. https:/www.aljazeera.com/news/2021/8/23/medigen-taiwan-rolls-out-local-covid-vaccinepresident-gets-jab

This article is made freely available for use in accordance with BMJ's website terms and conditions for the duration of the covid-19 pandemic or until otherwise determined by BMJ. You may use, download and print the article for any lawful, non-commercial purpose (including text and data mining) provided that all copyright notices and trade marks are retained. 